\title{
Polygenic influences associated with adolescent cognitive skills
}

\author{
Brittany L. Mitchell ${ }^{1,2^{*}}$, Narelle K. Hansell ${ }^{3}$, Kerrie McAloney ${ }^{1}$, Nicholas G. Martin ${ }^{1,2}$,
} Margaret J. Wright ${ }^{3,4}$, Miguel E. Renteria ${ }^{1,2}$, and Katrina L. Grasby ${ }^{1}$

1. Department of Genetics and Computational Biology, QIMR Berghofer Medical Research Institute, Brisbane, QLD, Australia

2. School of Biomedical Sciences, Faculty of Health, Queensland University of Technology (QUT), Brisbane, QLD, Australia.

3. Queensland Brain Institute, The University of Queensland, Brisbane, QLD, Australia.

4. Centre for Advanced Imaging, The University of Queensland, Brisbane, QLD, Australia.

* Corresponding author:

Brittany L. Mitchell

QIMR Berghofer Medical Research Institute

300 Herston Rd

Brisbane

4006

Australia

Brittany.mitchell@qimrberghofer.edu.au

Ph: +61 733620245

Keywords: polygenic risk scores; education; cognition; mental health; genetics; puberty;

adolescence; intelligence 


\begin{abstract}
Genes play an important role in children's cognitive ability through puberty and into adolescence. Recent advances in genomics has enabled us to test the effect of various genetic predispositions on measured cognitive outcomes. Here, we leveraged summary statistics from the most recent genome-wide association studies of eleven cognitive and mental health traits to build polygenic prediction models of measured intelligence and academic achievement in a cohort of Australian adolescent twins ( $\mathrm{N}=2,335,57 \%$ female). Additionally, we tested the association of these polygenic risk scores (PRS) with core academic skills such as the ability to comprehend, structure and sequence, evaluate and assess, communicate, and apply techniques and procedures. We show that PRSs for educational attainment, intelligence and cognitive factors explained up to $10 \%$ of the variance in educational achievement and $7 \%$ in intelligence test scores in our cohort. Additionally, we found that a genetic predisposition for ADHD was negatively associated with all cognitive outcomes and skills and a genetic predisposition for schizophrenia was negatively associated with performance IQ but no other cognitive domain. In this study, we show the potential value of genotypic data for predicting pupil achievement and cognitive developmental trajectory through puberty and into adolescence. We provide evidence that a genetic vulnerability to some mental health disorders is associated with poorer cognitive and educational outcomes, regardless of whether the individual has developed the disorder.
\end{abstract}




\section{Introduction}

It is well known that different people learn at different levels of ease and speed. Cognitive ability or intelligence can be broadly defined as an individual's ability to learn, reason, and infer when presented with new situations. In 1904, Charles Spearman first described a general intelligence factor, termed ' $\mathrm{g}$ ', to describe the observation where individuals that score highly in one type of intelligence test, will be likely to score high in others (positive inter-correlation) [1]. General intelligence can be measured through a variety of cognitive tests that output a standardized IQ score (Intelligence Quotient). Higher general intelligence is correlated with a wider range of variables, cognitive and other, than any other trait [2] including increased mortality [3], reduced fertility [4,5] and increased risk of mental and physical disease [6,7]. For more than a century, scientists have attempted to understand and identify factors that correlate with general intelligence.

A large body of literature has shown that cognitive ability is influenced by genetics and furthermore, that the influence of genetic factors on individual intelligence changes from childhood to adulthood [8-10]. Heritability estimates for intelligence increase dramatically as we age. Heritability is estimated to be $20 \%$ in childhood, $40 \%$ during adolescence and between $60-80 \%$ in adulthood [11-14]. Genome-wide association studies (GWAS) of cognitive ability have greatly increased our understanding of the genetic architecture underpinning these traits. To date, 206 independent variants have been associated with general intelligence (IQ) [15] in a meta-analysis of 270,000 individuals.

Different types or domains of intelligence are debated. Two commonly proposed types of intelligence are that of crystalized (the ability to learn and recall) and fluid (the ability to infer and reason in new situations) intelligence [16]. The widely used tests of intelligence developed by Wechsler assess performance in several domains, including verbal comprehension, nonverbal reasoning, working memory, and processing speed, which generally cluster together to provide an intelligence score [17]. Despite the identification of genetic variants associated with general intelligence, GWAS sample sizes are currently not large enough to detect unique genetic variation specific to cognitive domains.

The need for such large sample sizes is a result of the complex nature of intelligence as a genetic trait, meaning that it is influenced by the culmination of many genetic variants that each 
have small effect. A commonly used proxy for intelligence is that of educational attainment (EA) - the number of years of formal education. EA is correlated both phenotypically $(\sim 0.50)$ and genetically $(\sim 0.65)$ with intelligence $[11,18]$ but is regarded as a combination of both cognitive and non-cognitive factors, and is influenced by both genes and the environment $[19,20]$. Though a coarser measure than traditional intelligence tests, the highest level of education is a common question asked in almost all recruiting studies, and this has allowed researchers to rapidly gain large enough sample sizes to detect genetic variants (Single Nucleotide Polymorphisms; SNPs) with small effect. The most recent GWAS of EA (N= 1.1 million) identified 1,271 variants and estimated the heritability explained by common SNPs, i.e. the SNP-based heritability, to be 30\% [21]. Using the large GWAS of IQ, a recent study used genomic structural equation modelling (gSEM) to partition genetic variants associated with EA, the most well-powered cognitive trait, into two factors representing cognitive and non-cognitive components [22]. This novel approach has produced a well-powered proxy GWAS for the non-cognitive EA factor associated with educational attainment.

The wealth of genetic information produced from GWAS has led to the production of genetic predictors (polygenic risk scores; PRS). Polygenic risk scores have created the opportunity to operationalise an indicator of an individual's genetic predisposition for complex traits. Such studies have shown that PRS for intelligence or EA are able to significantly predict educational attainment [23], IQ test scores [24] as well as university entrance exam scores and university enrolment [25] in independent samples. Aligned with the increasing role of genetics in cognitive abilities during puberty and adolescents, PRS have been shown to account for a cumulatively larger proportion of variance in cognitive test scores and educational attainment between the ages of 7 and 16 [24,26]. To date, few studies have examined the domain or subject-specific genetic associations of cognitive ability. Phenotypically, education has been associated with improvement in particular domains of intellectual ability, such as working memory and vocabulary, rather than gains in general intelligence $[27,28]$. Several studies report high, but imperfect, genetic correlations $(\sim 60 \%)$ between achievement in reading and mathematics [29] as well as performance in various school subjects including English, art, mathematics and science [30]. Additionally, it has been shown that differences in EA genetic scores are associated with word reading ability [31] and reading comprehension [26]. Genetic variation associated with EA also differentially influences A-level subject choice and achievement in the United Kingdom [32] and has a stronger association with English rather than mathematics [33]. Although PRS are as yet imperfect measures of genetic predisposition, these studies 
demonstrate that PRS derived from GWAS of cognitive measures may be useful for differential statistical predication of domain-specific cognitive abilities and academic performance.

The Queensland Core Skills Test (QCST) is a test of academic achievement that was administered to the majority of Year 12 students in Queensland, Australia between 1992 and 2019. The test was used to assess individual achievement and core skills and the results were used for university entrance applications. Instead of testing subject specific knowledge, the QCST comprises a set of five 'baskets' that represent core academic skills. These baskets are aimed to assess higher order cognitive skills such as comprehension and communication, reasoning, and synthesis and integration of information. The QCST represents a unique resource to assess PRS associations of general academic achievement but also whether differential associations between academic skills exist [34].

Poorer cognitive performance is often associated with many mental health disorders, such as schizophrenia, bipolar and major depressive disorders, and has been shown to persist even after episodic and symptom remission [35,36]. In fact, a recent study reported that cognitive functions declined in individuals with psychotic disorders over the twenty years following their first hospitalization [37]. Impaired performance in specific cognitive domains has also been associated with mental health disorder status when compared to age-matched controls. Examples include decreased working memory, vocabulary and verbal fluency in individuals with schizophrenia [37,38] and deficits in working memory and executive function in individuals with depression [36]. However, after the onset of a mental health disorder, it is difficult to distinguish whether these observed associations are as a result of the subsequent environment or more innate and biological processes that precede disease onset.

The genetic relationship between cognitive ability and mental health disorders is also heterogeneous. Intelligence and EA have been found to have significant negative genetic correlations with schizophrenia $(-0.20)$, depressive symptoms $(-0.27)$, and attentiondeficit/hyperactivity disorder (ADHD) (-0.27) [39] but significant positive genetic correlations with bipolar (0.28) [40], autism (0.21) [39], and anorexia [41]. Despite these reported heterogeneous genetic associations between cognitive ability and mental health disorders, few studies have examined whether a genetic vulnerability to mental health disorders can negatively influence cognitive performance. While some studies have reported an association of a select PRS (such as schizophrenia or ADHD) with lower cognitive test scores $[42,43]$ in 
individuals that currently do not have a mental health disorder, no study has yet systematically examined this extensively across several mental health disorders and cognitive outcomes.

Here, we leveraged GWAS summary statistics from the most recent genome-wide association studies of intelligence, EA, cognitive and non-cognitive EA factors, as well as seven mental health disorders, to build prediction models of IQ test scores, academic skills, and academic achievement in a cohort of Australian adolescent twins.

\section{Methods}

\section{Participants}

A cohort of 2,335 adolescent twins (57\% female) from the Brisbane Longitudinal Twin study (BLTS) were used in these analyses [44]. Each individual completed the Multiple Aptitude Battery $(\mathrm{MAB})$ test at approximately the age of 16 (mean age $=16.6$, s.d. $=1.4)$. Each participant also completed a standardized test of academic achievement, the Queensland Core Skills Test (QCST), in August of their Year 12 of formal schooling (mean age $=17.4$, s.d. $=$ 0.4), for which we were granted consent to access their results. Standard genotyping and quality control procedures for the BLTS have been described previously [45]. Imputation was conducted using the Haplotype Reference Consortium 1.1 reference panel.

Written informed consent was obtained from each participant and from a parent or legal guardian for participants under the age of 18. The BLTS study was approved by the Human Research Ethics Committees of the QIMR Berghofer Medical Research Institute. Access to QCST results was approved by the Queensland Department of Education given participant and parent consent.

\section{Cognitive Performance Instruments}

Multidimensional Aptitude Battery (MAB)

The MAB, based on the Wechsler Adult Intelligence Scale (WAIS-R; Wechsler, 1981) [46], is a multiple-choice test of general intelligence that yields three scores: Verbal IQ, Performance IQ and Full-scale (VIQ, PIQ and FIQ respectively). FIQ is computed as a weighted sum of VIQ and PIQ. Verbal IQ is designed to measure an individual's ability to solve problems using language-based reasoning, which includes things such a vocabulary, arithmetic and general knowledge. Performance IQ is designed to measure visuospatial intellectual abilities and 
includes tasks like spatial arrangement, object assembly and attentiveness to detail. Further details regarding these subtests have been described previously $[47,48]$.

\section{Queensland Core Skills Test (QCST)}

Substantial additional detail regarding the QCST has been described previously (Wainwright et al., 2004, 2005). Briefly, the QCST is composed of five tests of core skills which are summed to give a total score that represents general academic achievement. These core skills, termed baskets, are as follows: Comprehend and Collect which entails comprehension of facts and interpretation to display meaning such as interpreting a data table. Structure and Sequence involves organizing and discerning patterns and relationships. Analyse, Assess and Conclude assesses the ability to deduce key messages and draw conclusions. Create and Present involves the use of written language to clearly present ideas and lastly, Apply Techniques and Procedures represents mathematical problem solving.

The total QCST score is scaled by the calendar year in which the test was written. However, individual baskets scores do not undergo any type of scaling. Therefore, we created residuals of the basket scores by removing the variance associated with which year the test was taken.

\section{Genetic Correlations and Polygenic Risk Score Calculation}

We estimated the pairwise genetic correlations between seven psychiatric disorders and four cognitive traits (Table 1) using linkage-disequilibrium score (ldsc) regression [49].

Table 1: Traits and their sources used for PGS construction using SBayesR

\begin{tabular}{|llcc|}
\hline Trait & Source & N cases & N controls \\
\hline Depression ${ }^{+}$ & Howard et al. 2019 & 246,819 & 561,485 \\
Anxiety & Purves et al. 2020 & 25,453 & 58,113 \\
Bipolar Disorder ${ }^{+}$ & Mullins et al. 2021 & 41,917 & 371,549 \\
Schizophrenia & Pardinas et al. 2018 & 40,675 & 64,643 \\
ADHD & Demontis et al. 2019 & 20,183 & 35,191 \\
Anorexia + & Watson et al. 2019 & 16,992 & 55,525 \\
Autism & Grove et al. 2019 & 18,381 & 27,969 \\
Educational Attainment + & Lee et al. 2018 & $1,100,000$ & NA \\
Intelligence ${ }^{+}$ & Savage et al. 2018 & 269,867 & NA \\
Cognitive skills & Demange et al. 2021 & $* 510,795$ & NA \\
Non-Cognitive skills & Demange et al. 2021 & $* 257,700$ & NA \\
\hline
\end{tabular}

* Sample sizes for the cognitive and non-cognitive skill factors are estimates using the output of genomicSEM. The traits themselves were not measured.

+ QIMR samples removed from GWAS summary statistics 
Only SNPs passing quality control (minor allele frequency $>0.01$, call rate $>0.9$ and imputation score > 0.6) were used for PRS calculation. Summary statistics for our 11 traits of interest in Table 1 were used for polygenic risk score calculation. Importantly, as participants at QIMR have participated in studies that were contributed to the several of these GWAS studies, PRS for these traits were calculated using summary statistics that excluded any QIMR cohorts. PRS were calculated using SBayesR, a Bayesian analysis was used to approximate the results of a conditional GWAS [50]. Polygenic risk scores were estimated by multiplying the multivariate effect size (obtained from SBayesR) times the allelic dosage of the effect allele and summing across all loci for each participant. For the LD reference, we used the same sparse LD matrix as used in Lloyd-Jones et al [50].

The effect of each PRS on the three IQ measures (performance, verbal, and full-scale) and on the six QCST measures (total and the 5 baskets) was estimated using a linear mixed model regression with the PRS as a predictor variable while accounting for sex, age and the first 10 genetic ancestry principal components included as covariates. Relatedness between participants was accounted for as a random effect with a genetic relatedness matrix, using GCTA 1.91 .7 [51,52]. A partial $\mathrm{R}^{2}$ was calculated and variance explained by the PRS was estimated as the difference in Pearson correlation coefficient between the full model (i.e. including the PRS) and a reduced model including only covariates. All significance values were corrected to account for multiple testing using FDR $<5 \%$.

\section{Results}

\section{Cohort descriptives}

Demographic information for the cohort used in this study is summarized in Table 2. 
Table 2: Summary of demographic and phenotypic measures of the BLTS cohort used in this study.

\begin{tabular}{|l|l|}
\hline & Mean (s.d.) [min - max] \\
\hline Age at IQ testing & $16.6(1.4)[15.7-28.9]$ \\
\hline Age at QCST & $19.9(0.4)[16.2-19.9]$ \\
\hline Sex & 1331 \\
Females (N) & 1002 \\
Males (N) & \\
\hline IQ Test Scores & $111.9(12.8)[77-153]$ \\
FIQ & $109.9(11.5)[77-153]$ \\
VIQ & $112.4(15.9)[64-151]$ \\
PIQ & \\
\hline QCST Test Scores & $176.9(26.7)[101.2-265]$ \\
Total QCST & $23.2(7)[5-44.5]$ \\
Comprehend and Collect & $20.4(6.7)[2.5-45]$ \\
Structure and Sequence & $25.4(8.1)[5.5-53.5]$ \\
Analyse, Assess and Conclude & $40.9(10.2)[1-73]$ \\
Create and Present & $14.5(6.7)[1-38.5]$ \\
Apply Techniques and Procedures &
\end{tabular}

\section{Genetic correlations between cognitive traits and mental health disorders}

While the genetic correlations among the mental health disorders were all positive, we found that the genetic correlations between our mental health and cognitive traits were highly heterogeneous (Figure 1). Anorexia and Autism were positively associated with EA, IQ as well as the cognitive and non-cognitive EA factors. Conversely, ADHD, depression and anxiety were negatively associated with these four traits (albeit, the correlation between depression and the cognitive skills factor did not survive multiple testing correction). We also observed several contrasting correlations with the cognitive and non-cognitive factors. For example, schizophrenia is negatively associated with IQ and the cognitive EA factor, but is positively correlated with the non-cognitive EA factor. Likewise, bipolar disorder is negatively correlated with cognitive EA factor, but positively correlated with EA and the non-cognitive EA factor (Figure 1). 


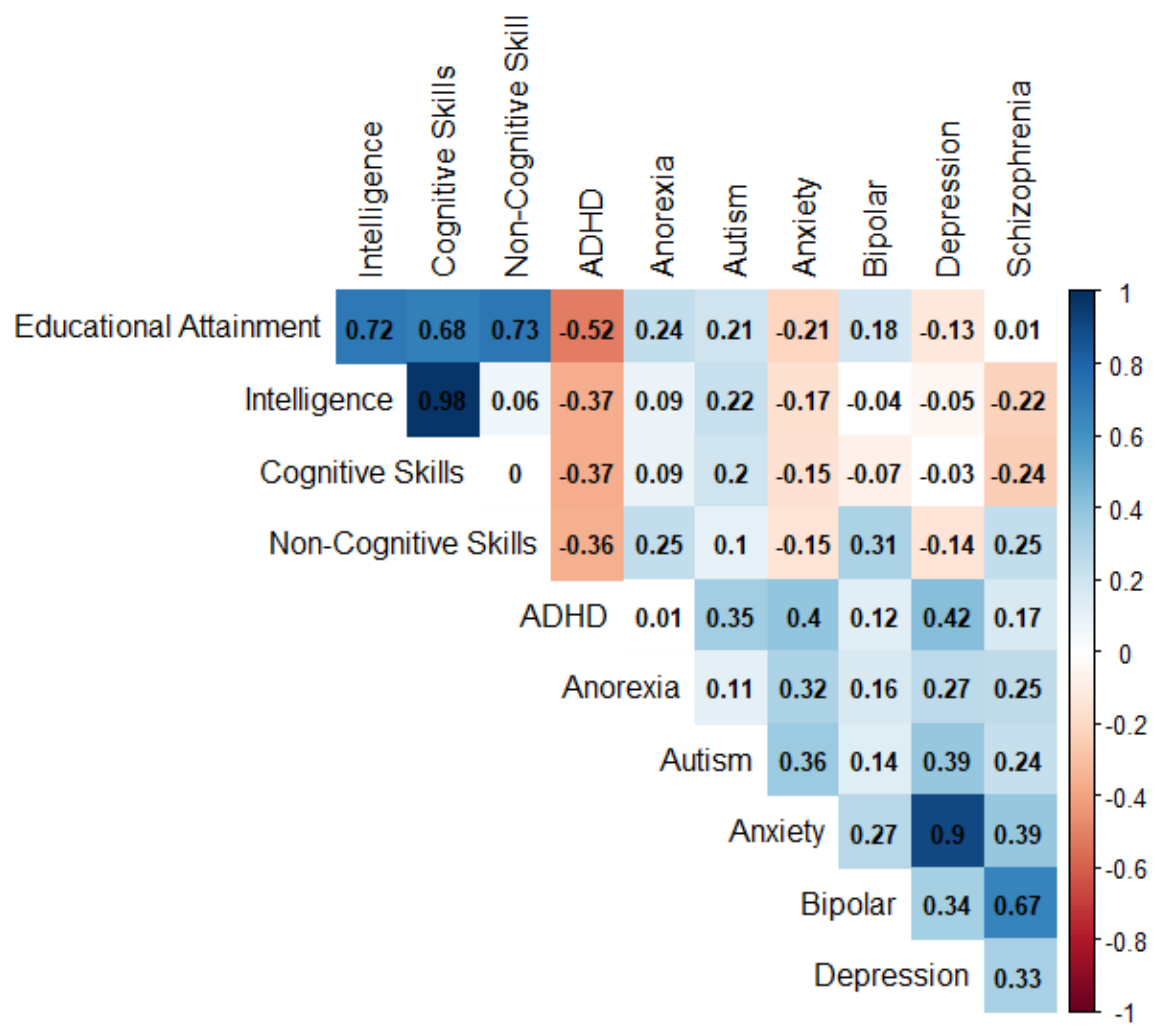

Figure 1: Genetic correlations between cognitive and psychiatric traits reveals highly heterogeneous genetic associations between mental health disorders and cognitive phenotypes.

\section{Cognitive and educational attainment PRS prediction of cognitive and academic skills}

A PRS for EA explained $10 \%$ of variance in total QCST scores $\left(\mathrm{p}=4.5 \times 10^{-40}\right), 4.5 \%$ in FIQ $\left(\mathrm{p}=7.9 \times 10^{-20}\right)$ and explained approximately $3 \mathrm{x}$ more variance in VIQ than PIQ $(6.8 \%$ and $2.1 \%$ respectively). Comparatively, a PRS for intelligence explained approximately $9.4 \%$ of variance in total QCST $\left(\mathrm{p}=4.8 \times 10^{-40}\right), 6.5 \%\left(\mathrm{p}=5.2 \times 10^{-27}\right)$ in VIQ and $4.8 \%\left(\mathrm{p}=2.2 \times 10^{-10}\right)$ in PIQ. The cognitive EA factor PRS had a highly similar association pattern to that of the IQ PRS. In contrast, the non-cognitive EA factor PRS only explained a small amount of variance in total $\operatorname{QCST}\left(2.2 \% ; \mathrm{p}=1.1 \times 10^{-9}\right)$, FIQ $(0.4 \% ; \mathrm{p}=0.008)$ and VIQ $\left(1.2 \% ; \mathrm{p}=4.9 \times 10^{-6}\right)$ and was not associated with PIQ ( $\mathrm{p}=0.51)$ (Figure 2). 


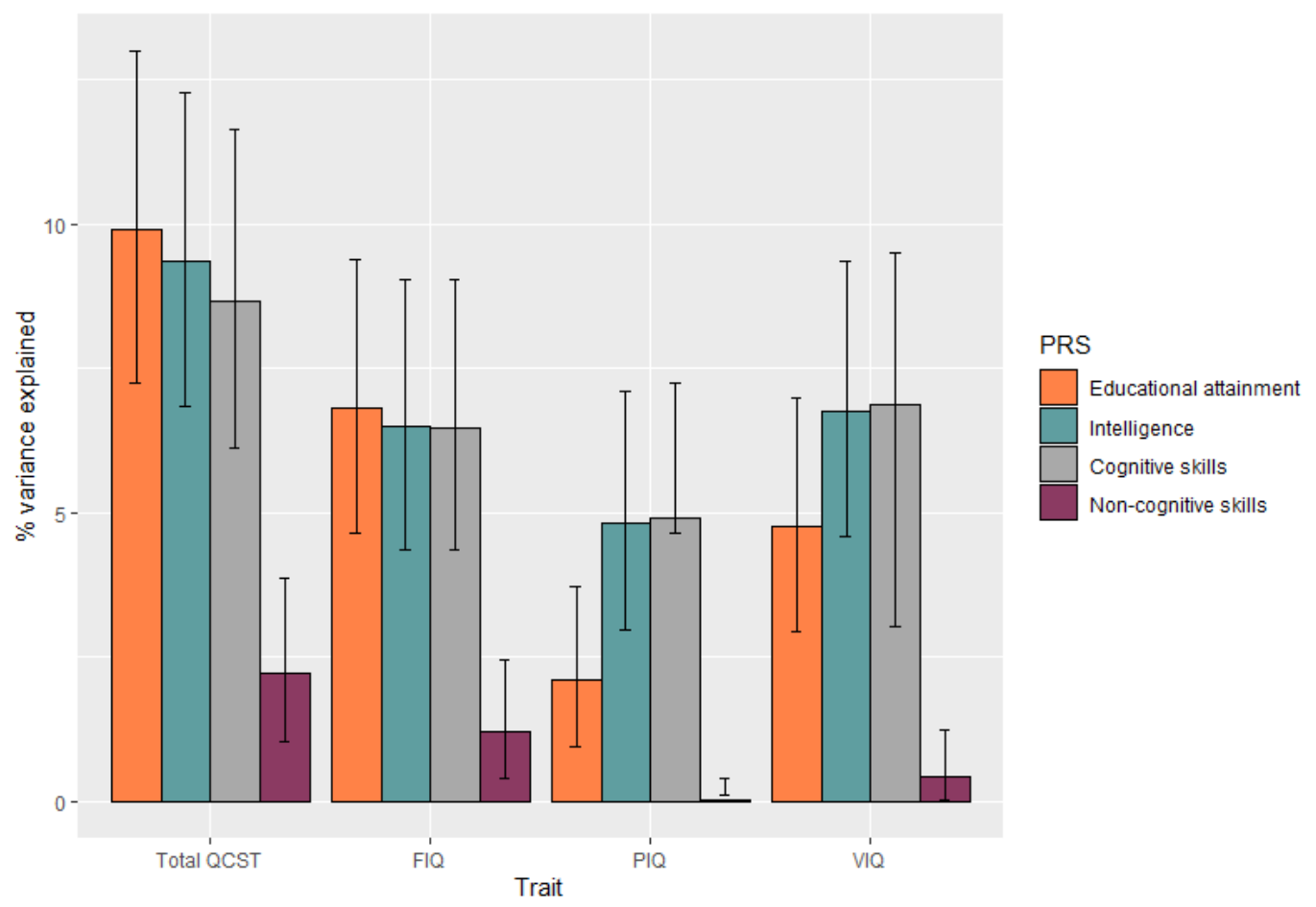

Figure 2: Barplot depicting percentage variance explained in total QCST, full IQ (FIQ), performance IQ (PIQ) and verbal IQ (VIQ) scores by the respective PRSs. PRS were constructed for educational attainment, intelligence, cognitive skills and non-cognitive skills.

Next, we tested the association between different QCST academic skills and the PRS from the four cognitive and educational attainment traits. Association results were largely similar across the different academic skills, indicating that a genetic predisposition to these cognitive and educational attainment traits does not differentially impact acquisition and performance of these academic skills (Figure 3).

\section{Mental Health Disorder PRS prediction of cognitive and academic skills}

Only the ADHD PRS was significantly associated with all IQ and academic measures whereby a higher genetic predisposition for ADHD is predictive of lower outcome scores. A genetic vulnerability to bipolar was negatively associated with PIQ and the academic skills 'comprehend and collect' as well as the 'application of techniques and procedures'. Several PRS showed association with only one cognitive outcome: depression and anxiety PRS were associated with lower scores for the academic skill 'applying techniques and procedures', and schizophrenia PRS was associated with lower performance IQ (Figure 3).

All remaining mental health PRS had non-significant associations with all IQ and academic measures, indicating that we did not find evidence that a genetic predisposition to these mental health disorders were associated with performance on these tests. 


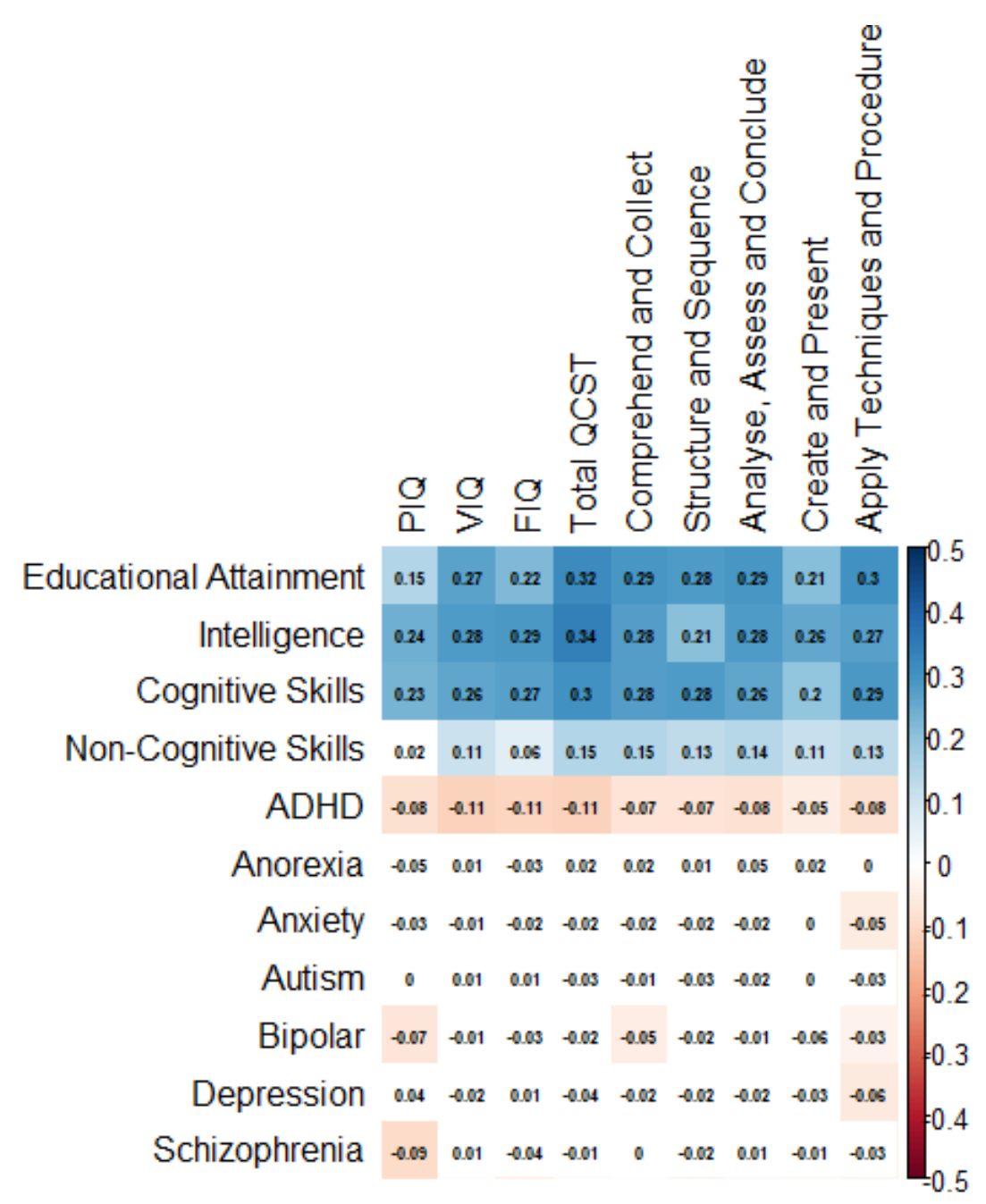

Figure 3: Fixed effects of association results between PRS and cognitive outcomes. Associations in white did not survive multiple testing correction (FDR $<5 \%)$

\section{Discussion}

Intelligence is important to both individuals and society as a whole. Understanding the mechanisms influencing intellectual ability is crucial to better understand relationships between cognitive abilities and psychosocial, educational and economic outcomes. This study had two main objectives, first, to test the association between PRS for EA, intelligence, the cognitive and non-cognitive EA factors and IQ scores, as well as unique measures of academic skills assessed during the QCST. Second, we explored the associations between genetic predispositions to seven mental health disorders and the same cognitive and academic scores as above. 
In line with previous findings, we found that individuals with higher PRS for EA, intelligence and the cognitive EA factor performed better in all IQ and academic tests. Interestingly, PRSs for EA and intelligence explained roughly the same amount of variance in total QCST and fullscale IQ test scores, despite the greater statistical power of the EA GWAS. However, while EA may be a good proxy for full-scale IQ, it did not perform as well for verbal and performance IQ, which were better predicted by intelligence PRS or cognitive EA factor PRS. In fact, intelligence PRS explained approximately twice as much variance in performance IQ than EA PRS. PRS for the non-cognitive EA factor significantly predicted total QCST scores, as well as the five individual core academic skills, albeit more weakly than the cognitive EA factor. This indicates that there are genetic influences independent of intelligence that contribute to higher educational attainment that also capture variance in these higher-order academic skills. When it comes to intelligence scores, it appears that genetic influences on the cognitive component of educational attainment contribute almost equally to both performance and verbal IQ. Interestingly, genetic influences on the non-cognitive EA factor (i.e. independent of intelligence) contribute to verbal IQ. This probably results from using a full-scale IQ or first principal component in the GWAS on intelligence, which does not fully capture the genetic influences on each of the performance and verbal IQ domains. It suggests that if there are unique genetic influences on these IQ domains, those influencing educational attainment are more strongly associated with verbal IQ than performance IQ.

Cognitive impairment is common in individuals living with mental health disorders. However, several studies have shown evidence that cognitive impairment exists before the onset of disorders such as schizophrenia [53,54] and, to a lesser extent, bipolar [55]. Additionally, it has also been shown that cognitive ability is impaired in relatives of those with both schizophrenia and bipolar disorder [56-59]. These findings suggest both a genetic contribution to cognitive impairment in these disorders and that cognitive ability may be influenced by factors that are correlated with increased liability to the disorder. With the advances in GWAS studies, we have been able to explore genetic associations between cognitive phenotypes and mental health disorders. While it has been shown that PRS for cognitive phenotypes are able to predict cognitive ability during adolescence, it has also been shown that a genetic vulnerability to mental health disorders can negatively influence cognitive performance, despite the absence of the actual disorder. For example, a higher PRS for schizophrenia has been associated with lower performance IQ and language fluency [60] and a higher PRS for ADHD has been associated decreased working memory in children aged 7-11 [61]. 
We found that a genetic predisposition for ADHD was negatively associated with all IQ and academic outcomes, despite the limited statistical power of the ADHD PRS compared to other mental health traits. These findings are in line with previous studies that found PRS for ADHD were associated with lower educational and IQ test scores in a sample of 6,900 adolescents in the UK [62] and studies where PRS for EA and IQ predicted ADHD traits [2,63]. Our results demonstrate the pervasiveness of this association across five academic skill domains that are rarely measured in tests of achievement and are representative of higher-order skills that are thought to generalise across academic subjects. These findings point to genetic overlap between ADHD and cognitive abilities and highlight the importance of recognizing that ADHD risk may functionally contribute to poorer cognitive outcomes across numerous domains.

Although cognitive impairment is a frequent observation in those at risk of schizophrenia, only the association between schizophrenia PRS and performance IQ maintained statistical significance after multiple testing correction. Our results replicate previous reports of an association between schizophrenia and adolescent performance IQ on both the phenotypic level [64] and using genetic risk scores [60], and supports evidence of impaired spatial functioning in individuals with schizophrenia [65] or with a genetic vulnerability for schizophrenia [66]. Together, these findings suggest that specific domains, namely performance or fluid intelligence, may be compromised in individuals at higher genetic risk of schizophrenia rather than general intellectual ability. In a similar vein, a genetic predisposition was not associated with compromised performance in the higher-order academic skills assessed with the QCST.

A genetic vulnerability for bipolar disorder was also negatively associated with performance IQ, perhaps driven by the high genetic correlation between bipolar disorder and schizophrenia. None of the remaining mental health trait PRSs had significant associations with IQ measures or total QCST scores. However, ADHD, anxiety, depression and bipolar PrS were all negatively associated with the Applying Techniques and Procedures basket. This basket tests the academic skill of information integration and problem-solving [67]. Bipolar disorder PRS was also significantly negatively associated with the Comprehend and Collect QCST basket. Together this points towards domain-specific impairment in individuals with genetic risk for these disorders, and sheds light on possible mechanisms underlying the observed relationship between these disorders and impaired cognitive ability. 
It is important to note that PRS associations do not necessarily imply causality but rather highlight how genetic relationships and associations express in phenotypic variability. However, a causal relationship between intelligence and some of these mental health traits has been demonstrated previously in the latest IQ GWAS [15]. The authors used Mendelian Randomization to assess the causal relationships of some of these observations and found a significant protective effect of intelligence on schizophrenia and ADHD and intelligence was shown to be a risk factor for autism. In contrast, we find no significant support of a PRS for autism affecting cognitive test scores and the direction of association is predominantly negative. Lastly, despite significant genetic correlations, we found no significant influence of PRS for anorexia or autism on our cognitive outcomes, though this might be because of limited statistical power rather than the absence of a true effect.

Our study has several limitations. First, the GWASs used to create the PRS only explain a small fraction of variance in each respective trait in the general population. Additionally, the statistical power of each GWAS is substantially different and therefore the absence of a significant association for some traits may be due to lack of power and not the absence of a true association. Additionally, the QCST participants may be regarded as a biased sample of the cohort because less academically able students escape ascertainment by not sitting the test. While we have attempted to account for this through the inclusion of IQ measures, it is possible these individuals are also not truly representative of the general population given biases involved in the voluntary participation in research studies.

Overall, our results support the hypothesis of a general cognitive factor for which the genetic underpinnings are being measured through various proxies in GWAS studies. Additionally, we show that cognitive impairment due to biological risk of mental health disorders may be observable in the general population from an early age, before the onset of any adult forms of psychopathology and may effect individual developmental trajectory.

\section{Acknowledgments}

We greatly appreciate the support from the twins and their parents and their willingness to participate in this study. We are grateful to Marlene Grace, Ann Eldridge and Kathleen Moore 
for recruitment of twin pairs and data collection. We also thank the QSA (formerly QSSSSB) for permission to use their data and their ongoing assistance with data retrieval.

\section{Funding}

This project was supported by grants from the Australian Research Council (Grant Numbers. A 79600334, A 79906588, A 79801419), and the Human Frontier Science Program (Grant Number RG0154/1998B). KLG was supported by APP1173025. BLM is grateful for support from Queensland University of Technology through a QUT Postgraduate Research Scholarship.

\section{Author Contributions}

B.L.M and K.L.G designed and supervised the study. B.L.M performed the analyses, with support and input from K.L.G. and M.E.R . M.J.W., N.K.H., K.M. and N.G.M. were responsible for the cohort design, genotyping, and data acquisition, processing and quality control. B.L.M. wrote the first draft of the manuscript, and integrated input and feedback from all co-authors. All authors read and approved the final manuscript.

\section{Conflicts of Interest}

All authors report no conflicts of interest in relation with this study. 


\section{References}

1. Spearman, C. " General Intelligence," objectively determined and measured. The American Journal of Psychology 1904, 15, 201-292.

2. Krapohl, E.; Euesden, J.; Zabaneh, D.; Pingault, J.; Rimfeld, K.; Von Stumm, S.; Dale, P.; Breen, G.; O'Reilly, P.F.; Plomin, R. Phenome-wide analysis of genome-wide polygenic scores. Mol. Psychiatry 2016, 21, 1188-1193.

3. Batty, G.D.; Deary, I.J.; Gottfredson, L.S. Premorbid (early life) IQ and later mortality risk: systematic review. Ann. Epidemiol. 2007, 17, 278-288.

4. $\quad$ Barban, N.; Jansen, R.; De Vlaming, R.; Vaez, A.; Mandemakers, J.J.; Tropf, F.C.; Shen, X.; Wilson, J.F.; Chasman, D.I.; Nolte, I.M. Genomewide analysis identifies 12 loci influencing human reproductive behavior. Nat. Genet. 2016, 48, 1462.

5. Day, F.R.; Helgason, H.; Chasman, D.I.; Rose, L.M.; Loh, P.-R.; Scott, R.A.; Helgason, A.; Kong, A.; Masson, G.; Magnusson, O.T. Physical and neurobehavioral determinants of reproductive onset and success. Nat. Genet. 2016, 48, 617.

6. Hill, W.D.; Arslan, R.C.; Xia, C.; Luciano, M.; Amador, C.; Navarro, P.; Hayward, C.; Nagy, R.; Porteous, D.J.; McIntosh, A.M. Genomic analysis of family data reveals additional genetic effects on intelligence and personality. Mol. Psychiatry 2018, 23, 2347.

7. Deary, I.J.; Strand, S.; Smith, P.; Fernandes, C. Intelligence and educational achievement. Intelligence 2007, 35, 13-21.

8. Von Stumm, S.; Plomin, R. Socioeconomic status and the growth of intelligence from infancy through adolescence. Intelligence 2015, 48, 30-36.

9. $\quad$ Bartels, M.; Rietveld, M.J.; Van Baal, G.C.M.; Boomsma, D.I. Heritability of educational achievement in 12-year-olds and the overlap with cognitive ability. Twin Research and Human Genetics 2002, 5, 544553.

10. Deary, I.J.; Whalley, L.J.; Lemmon, H.; Crawford, J.; Starr, J.M. The stability of individual differences in mental ability from childhood to old age: follow-up of the 1932 Scottish Mental Survey. Intelligence 2000, 28, 49-55.

11. Plomin, R.; von Stumm, S. The new genetics of intelligence. Nature Reviews Genetics 2018, 19, 148.

12. Haworth, C.M.; Wright, M.J.; Martin, N.W.; Martin, N.G.; Boomsma, D.I.; Bartels, M.; Posthuma, D.; Davis, O.S.; Brant, A.M.; Corley, R.P. A twin 
study of the genetics of high cognitive ability selected from 11,000 twin pairs in six studies from four countries. Behav. Genet. 2009, 39, 359-370.

13. Hansell, N.; Wright, M.; Luciano, M.; Geffen, G.; Geffen, L.; Martin, N. Genetic covariation between event-related potential (ERP) and behavioral non-ERP measures of working-memory, processing speed, and IQ. Behav. Genet. 2005, 35, 695-706.

14. Finkel, D.; Pedersen, N.; McGue, M. Genetic influences on memory performance in adulthood: comparison of Minnesota and Swedish twin data. Psychol. Aging 1995, 10, 437.

15. Savage, J.E.; Jansen, P.R.; Stringer, S.; Watanabe, K.; Bryois, J.; De Leeuw, C.A.; Nagel, M.; Awasthi, S.; Barr, P.B.; Coleman, J.R. Genomewide association meta-analysis in 269,867 individuals identifies new genetic and functional links to intelligence. Nat. Genet. 2018, 50, 912.

16. Cattell, R.B. Theory of fluid and crystallized intelligence: A critical experiment. J. Educ. Psychol. 1963, 54, 1.

17. Wechsler, D. Wechsler adult intelligence scale. Arch. Clin. Neuropsychol. 1955.

18. Rietveld, C.A.; Esko, T.; Davies, G.; Pers, T.H.; Turley, P.; Benyamin, B.; Chabris, C.F.; Emilsson, V.; Johnson, A.D.; Lee, J.J. Common genetic variants associated with cognitive performance identified using the proxy-phenotype method. Proceedings of the National Academy of Sciences 2014, 111, 13790-13794.

19. Krapohl, E.; Plomin, R. Genetic link between family socioeconomic status and children's educational achievement estimated from genome-wide SNPs. Mol. Psychiatry 2016, 21, 437.

20. Belsky, D.W.; Domingue, B.W.; Wedow, R.; Arseneault, L.; Boardman, J.D.; Caspi, A.; Conley, D.; Fletcher, J.M.; Freese, J.; Herd, P. Genetic analysis of social-class mobility in five longitudinal studies. Proceedings of the National Academy of Sciences 2018, 115, E7275E7284.

21. Lee, J.J.; Wedow, R.; Okbay, A.; Kong, E.; Maghzian, O.; Zacher, M.; Nguyen-Viet, T.A.; Bowers, P.; Sidorenko, J.; Linnér, R.K. Gene discovery and polygenic prediction from a genome-wide association study of educational attainment in 1.1 million individuals. Nat. Genet. 2018, 50, 1112.

22. Demange, P.A.; Malanchini, M.; Mallard, T.T.; Biroli, P.; Cox, S.R.; Grotzinger, A.D.; Tucker-Drob, E.M.; Abdellaoui, A.; Arseneault, L.; Van Bergen, E. Investigating the genetic architecture of noncognitive skills using GWAS-by-subtraction. Nat. Genet. 2021, 53, 35-44. 
23. Selzam, S.; Krapohl, E.; von Stumm, S.; O'reilly, P.; Rimfeld, K.; Kovas, Y.; Dale, P.; Lee, J.; Plomin, R. Predicting educational achievement from DNA. Mol. Psychiatry 2017, 22, 267.

24. Allegrini, A.G.; Selzam, S.; Rimfeld, K.; von Stumm, S.; Pingault, J.-B.; Plomin, R. Genomic prediction of cognitive traits in childhood and adolescence. Mol. Psychiatry 2019, 24, 819-827.

25. Smith-Woolley, E.; Ayorech, Z.; Dale, P.S.; von Stumm, S.; Plomin, R. The genetics of university success. Sci. Rep. 2018, 8, 14579.

26. Selzam, S.; Dale, P.S.; Wagner, R.K.; DeFries, J.C.; Cederlöf, M.; O’Reilly, P.F.; Krapohl, E.; Plomin, R. Genome-wide polygenic scores predict reading performance throughout the school years. Scientific Studies of Reading 2017, 21, 334-349.

27. Ritchie, S.J.; Bates, T.C.; Deary, I.J. Is education associated with improvements in general cognitive ability, or in specific skills? Dev. Psychol. 2015, 51, 573.

28. Melby-Lervåg, M.; Hulme, C. Is working memory training effective? A meta-analytic review. Dev. Psychol. 2013, 49, 270.

29. Davis, O.S.; Band, G.; Pirinen, M.; Haworth, C.M.; Meaburn, E.L.; Kovas, Y.; Harlaar, N.; Docherty, S.J.; Hanscombe, K.B.; Trzaskowski, M. The correlation between reading and mathematics ability at age twelve has a substantial genetic component. Nature Communications 2014, 5, 1-6.

30. Rimfeld, K.; Kovas, Y.; Dale, P.S.; Plomin, R. Pleiotropy across academic subjects at the end of compulsory education. Sci. Rep. 2015, 5, 1-12.

31. Belsky, D.W.; Moffitt, T.E.; Corcoran, D.L.; Domingue, B.; Harrington, H.; Hogan, S.; Houts, R.; Ramrakha, S.; Sugden, K.; Williams, B.S. The genetics of success: How single-nucleotide polymorphisms associated with educational attainment relate to life-course development. Psychol. Sci. 2016, 27, 957-972.

32. Rimfeld, K.; Ayorech, Z.; Dale, P.S.; Kovas, Y.; Plomin, R. Genetics affects choice of academic subjects as well as achievement. Sci. Rep. 2016, 6, 1-9.

33. Ward, M.E.; McMahon, G.; St Pourcain, B.; Evans, D.M.; Rietveld, C.A.; Benjamin, D.J.; Koellinger, P.D.; Cesarini, D.; Consortium, S.S.G.A.; Smith, G.D. Genetic variation associated with differential educational attainment in adults has anticipated associations with school performance in children. PLoS One 2014, 9, e100248.

34. Authority, Q.S. All you need to know about the Queensland Core Skills Test. 2003.

35. Robinson, L.J.; Thompson, J.M.; Gallagher, P.; Goswami, U.; Young, A.H.; Ferrier, I.N.; Moore, P.B. A meta-analysis of cognitive deficits in 
euthymic patients with bipolar disorder. J. Affect. Disord. 2006, 93, 105-115.

36. Rock, P.; Roiser, J.; Riedel, W.; Blackwell, A. Cognitive impairment in depression: a systematic review and meta-analysis. Psychol. Med. 2014, 44, 2029-2040.

37. Fett, A.-K.J.; Velthorst, E.; Reichenberg, A.; Ruggero, C.J.; Callahan, J.L.; Fochtmann, L.J.; Carlson, G.A.; Perlman, G.; Bromet, E.J.; Kotov, R. Long-term changes in cognitive functioning in individuals with psychotic disorders: findings from the Suffolk County Mental Health Project. JAMA Psychiatry 2020, 77, 387-396.

38. Trivedi, J.K. Cognitive deficits in psychiatric disorders: Current status. Indian J. Psychiatry 2006, 48, 10.

39. Sniekers, S.; Stringer, S.; Watanabe, K.; Jansen, P.R.; Coleman, J.R.; Krapohl, E.; Taskesen, E.; Hammerschlag, A.R.; Okbay, A.; Zabaneh, D. Genome-wide association meta-analysis of 78,308 individuals identifies new loci and genes influencing human intelligence. Nat. Genet. 2017, 49, 1107.

40. Okbay, A.; Beauchamp, J.P.; Fontana, M.A.; Lee, J.J.; Pers, T.H.; Rietveld, C.A.; Turley, P.; Chen, G.-B.; Emilsson, V.; Meddens, S.F.W. Genomewide association study identifies 74 loci associated with educational attainment. Nature 2016, 533, 539.

41. Watson, H.J.; Yilmaz, Z.; Thornton, L.M.; Hübel, C.; Coleman, J.R.; Gaspar, H.A.; Bryois, J.; Hinney, A.; Leppä, V.M.; Mattheisen, M. Genome-wide association study identifies eight risk loci and implicates metabo-psychiatric origins for anorexia nervosa. Nat. Genet. 2019, 51, 1207-1214.

42. Shafee, R.; Nanda, P.; Padmanabhan, J.L.; Tandon, N.; AllieyRodriguez, N.; Kalapurakkel, S.; Weiner, D.J.; Gur, R.E.; Keefe, R.S.; Hill, S.K. Polygenic risk for schizophrenia and measured domains of cognition in individuals with psychosis and controls. Translational Psychiatry 2018, 8, 1-9.

43. Dickinson, D.; Zaidman, S.R.; Giangrande, E.J.; Eisenberg, D.P.; Gregory, M.D.; Berman, K.F. Distinct polygenic score profiles in schizophrenia subgroups with different trajectories of cognitive development. Am. J. Psychiatry 2020, 177, 298-307.

44. Wright, M.J.; Martin, N.G. Brisbane adolescent twin study: outline of study methods and research projects. Aust. J. Psychol. 2004, 56, 6578.

45. Colodro-Conde, L.; Couvy-Duchesne, B.; Zhu, G.; Coventry, W.L.; Byrne, E.M.; Gordon, S.; Wright, M.J.; Montgomery, G.W.; Madden, P.A.; Ripke, S. A direct test of the diathesis-stress model for depression. Mol. Psychiatry 2018, 23, 1590-1596. 
46. Jackson, D.N. Multidimensional aptitude battery II: manual; Sigma Assessment Systems Port Huron, MI: 1998.

47. Luciano, M.; Wright, M.; Smith, G.; Geffen, G.; Geffen, L.; Martin, N. Genetic covariance among measures of information processing speed, working memory, and IQ. Behav. Genet. 2001, 31, 581-592.

48. Wainwright, M.A.; Wright, M.J.; Geffen, G.M.; Luciano, M.; Martin, N.G. The genetic basis of academic achievement on the Queensland Core Skills Test and its shared genetic variance with IQ. Behav. Genet. 2005, 35, 133-145.

49. Bulik-Sullivan, B.; Finucane, H.K.; Anttila, V.; Gusev, A.; Day, F.R.; Loh, P.R.; ReproGen, C.; Psychiatric Genomics, C.; Genetic Consortium for Anorexia Nervosa of the Wellcome Trust Case Control, C.; Duncan, L.; et al. An atlas of genetic correlations across human diseases and traits. Nat. Genet. 2015, 47, 1236-1241, doi:10.1038/ng.3406.

50. Lloyd-Jones, L.R.; Zeng, J.; Sidorenko, J.; Yengo, L.; Moser, G.; Kemper, K.E.; Wang, H.; Zheng, Z.; Magi, R.; Esko, T. Improved polygenic prediction by Bayesian multiple regression on summary statistics. Nature Communications 2019, 10, 1-11.

51. Yang, J.; Zaitlen, N.A.; Goddard, M.E.; Visscher, P.M.; Price, A.L. Advantages and pitfalls in the application of mixed-model association methods. Nat. Genet. 2014, 46, 100.

52. Yang, J.; Lee, S.H.; Goddard, M.E.; Visscher, P.M. GCTA: a tool for genome-wide complex trait analysis. The American Journal of Human Genetics 2011, 88, 76-82.

53. Khandaker, G.M.; Barnett, J.H.; White, I.R.; Jones, P.B. A quantitative meta-analysis of population-based studies of premorbid intelligence and schizophrenia. Schizophr. Res. 2011, 132, 220-227.

54. Keefe, R.S.; Fenton, W.S. How should DSM-V criteria for schizophrenia include cognitive impairment? Schizophr. Bull. 2007, 33, 912-920.

55. Bora, E. Developmental trajectory of cognitive impairment in bipolar disorder: comparison with schizophrenia. Eur. Neuropsychopharmacol. 2015, 25, 158-168.

56. Doyle, A.E.; Wozniak, J.; Wilens, T.E.; Henin, A.; Seidman, L.J.; Petty, C.; Fried, R.; Gross, L.M.; Faraone, S.V.; Biederman, J. Neurocognitive impairment in unaffected siblings of youth with bipolar disorder. Psychol. Med. 2009, 39, 1253-1263.

57. Klimes-Dougan, B.; Ronsaville, D.; Wiggs, E.A.; Martinez, P.E. Neuropsychological functioning in adolescent children of mothers with a history of bipolar or major depressive disorders. Biol. Psychiatry 2006, 60, 957-965.

58. Bora, E.; Lin, A.; Wood, S.; Yung, A.; McGorry, P.; Pantelis, C. Cognitive deficits in youth with familial and clinical high risk to psychosis: $\mathrm{a}$ 
systematic review and meta-analysis. Acta Psychiatr. Scand. 2014, 130, 1-15.

59. Seidman, L.J.; Hellemann, G.; Nuechterlein, K.H.; Greenwood, T.A.; Braff, D.L.; Cadenhead, K.S.; Calkins, M.E.; Freedman, R.; Gur, R.E.; Gur, R.C. Factor structure and heritability of endophenotypes in schizophrenia: findings from the Consortium on the Genetics of Schizophrenia (COGS-1). Schizophr. Res. 2015, 163, 73-79.

60. Riglin, L.; Collishaw, S.; Richards, A.; Thapar, A.K.; Maughan, B.; O'Donovan, M.C.; Thapar, A. Schizophrenia risk alleles and neurodevelopmental outcomes in childhood: a population-based cohort study. The Lancet Psychiatry 2017, 4, 57-62.

61. Aguilar-Lacasaña, S.; Vilor-Tejedor, N.; Jansen, P.R.; López-Vicente, M.; Bustamante, M.; Burgaleta, M.; Sunyer, J.; Alemany, S. Polygenic risk for ADHD and ASD and their relation with cognitive measures in school children. Psychol. Med. 2020, 1-9.

62. Stergiakouli, E.; Martin, J.; Hamshere, M.L.; Heron, J.; St Pourcain, B.; Timpson, N.J.; Thapar, A.; Davey Smith, G. Association between polygenic risk scores for attention-deficit hyperactivity disorder and educational and cognitive outcomes in the general population. Int. J. Epidemiol. 2017, 46, 421-428.

63. de Zeeuw, E.L.; van Beijsterveldt, C.E.; Glasner, T.J.; Bartels, M.; Ehli, E.A.; Davies, G.E.; Hudziak, J.J.; Consortium, S.S.G.A.; Rietveld, C.A.; Groen-Blokhuis, M.M. Polygenic scores associated with educational attainment in adults predict educational achievement and ADHD symptoms in children. American Journal of Medical Genetics Part B: Neuropsychiatric Genetics 2014, 165, 510-520.

64. Hubbard, L.; Tansey, K.E.; Rai, D.; Jones, P.; Ripke, S.; Chambert, K.D.; Moran, J.L.; McCarroll, S.A.; Linden, D.E.; Owen, M.J. Evidence of common genetic overlap between schizophrenia and cognition. Schizophr. Bull. 2016, 42, 832-842.

65. Piskulic, D.; Olver, J.S.; Norman, T.R.; Maruff, P. Behavioural studies of spatial working memory dysfunction in schizophrenia: a quantitative literature review. Psychiatry Res. 2007, 150, 111-121.

66. Glahn, D.C.; Therman, S.; Manninen, M.; Huttunen, M.; Kaprio, J.; Lönnqvist, J.; Cannon, T.D. Spatial working memory as an endophenotype for schizophrenia. Biol. Psychiatry 2003, 53, 624-626.

67. Wainwright, M.A.; Wright, M.J.; Luciano, M.; Geffen, G.M.; Martin, N.G. Multivariate genetic analysis of academic skills of the Queensland core skills test and IQ highlight the importance of genetic g. Twin Research and Human Genetics 2005, 8, 602-608. 